\title{
Applications of Fuzzy Control in Greenhouse Intelligent Control System
}

\author{
Jin Yao
}

School Department of mechanics and materials engineering, Wuzhou University, Wuzhou 543000, China

Keywords: fuzzy control; Fuzzy rules; simulation.

\begin{abstract}
Given the greenhouse microenvironment is more than one variable, strong coupling, large delay, time-varying controlled object, unable to establish an accurate mathematical model, the conventional switch or PID control cannot obtain the desired control effect, this paper results of previous studies lessons based on the design of the fuzzy controller based on fuzzy control theory intelligence. This paper studies the application of fuzzy control in greenhouse environment control of, developed a greenhouse intelligent control system, and has achieved good control effect in practical applications.
\end{abstract}

\section{Introduction}

Fuzzy control is an important branch of intelligent control of the core, it does not depend on accurate mathematical model of the controlled system, but through qualitative understanding of the dynamic characteristics of the system, a direct inference to determine or change the on-line control strategies to achieve complex, nonlinear uncertainty accused the control system, this method is easy to achieve, but also easier to ensure real-time nature. Fuzzy control is highly intelligent humanoid features, it does not depend on the characteristics of precise mathematical model and has strong robustness and adaptability is an effective way to solve complex control problems. This chapter will compare the differences of fuzzy control system and the traditional control systems, this paper introduces the core of the fuzzy control system - the principle of fuzzy controller, structure, design, content and design methods.

\section{The basic composition and control principle of fuzzy control system}

Three core components of traditional control systems is the controller, the controlled object and feedback sensing channel. Assume that the system is part of the output of each of its inputs and (or difference) of the linear function, there are:

$$
\begin{aligned}
& y=G\left(x_{2}\right)=G(d+u) \\
& v=H\left(x_{3}\right)=H(y+n) \\
& u=C\left(x_{1}\right)=C(r-v)
\end{aligned}
$$

Thus the equation can be written summing the respective points as follows:

$$
\left\{\begin{array} { l } 
{ x _ { 1 } = r - v = r - H x _ { 3 } } \\
{ x _ { 2 } = d + u = d + G x _ { 1 } } \\
{ x _ { 3 } = y + n = n + G x _ { 2 } }
\end{array} , \text { Namely } \left\{\begin{array}{l}
x_{1}+H x_{3}=r \\
-G x_{1}+x_{2}=d \\
G x_{2}+x_{3}=n
\end{array}\right.\right.
$$

Its matrix equation is:

$$
\left[\begin{array}{ccc}
1 & 0 & H \\
-C & 1 & 0 \\
0 & -G & 1
\end{array}\right]\left[\begin{array}{l}
x_{1} \\
x_{2} \\
x_{3}
\end{array}\right]=\left[\begin{array}{l}
r \\
d \\
n
\end{array}\right]
$$

So we can obtain:

$$
\left[\begin{array}{l}
x_{1} \\
x_{2} \\
x_{3}
\end{array}\right]=\frac{1}{1+G C H}\left[\begin{array}{ccc}
1 & -G H & -H \\
C & 1 & -C H \\
G C & G & 1
\end{array}\right]\left[\begin{array}{l}
r \\
d \\
n
\end{array}\right]
$$

These are the traditional control systems analysis and design, especially the main basis for the design of the controller $\mathrm{C}$. The control of the target system is controlled by adjusting the input signal 
$\mathrm{u}, \mathrm{y}$ to achieve compliance with the output signal form required, or that the difference (r-y) as small as possible, i.e., by adjusting the output by as near the signal $r$.

\section{Comparison of models}

The basic operating principle of fuzzy control system are as follows: First, take the system through sensor data acquisition unit controlled variable, conversion and arithmetic processing, the exact value of the output, and then with a given value, to obtain accurate bias, and then through the fuzzy controller the fuzzy processing, fuzzy rules and inference operation, and finally by the exact amount of the fine processing output, through D/A converted analog actuator to control the controlled object in order to achieve the purpose. Common "Honest" (Positive Big, PB), "middle" (Positive Middle, PM), "are small" (Positive Small, PS), "Zero" (Zero, 0), "negative small" (Negative Small, NS), "negative in" (Negative Middle, NM), "big negative" (Negative Big, NB) to represent. Divided unrestricted linguistic variables can be arbitrarily divided into several values, mainly divided according to different fuzzy reasoning.

The quantization error e $\mathrm{k}$ factor is defined as:

$$
k_{e} \stackrel{\text { def }}{=} \frac{n}{e}
$$

Enter the amount of the error e of the basic domain of [-e, e], $n$ is the basic theory to quantify the number of domain file. Quantization factor is defined as the rate of change of the error:

$$
k_{e c} \stackrel{\operatorname{def}}{=} \frac{n}{e c}
$$

Input error rate of change basic domain [etc., etc.], $\mathrm{n}$ is the number of basic domain quantify file. The scale factor controls the amount of change is defined as:

$$
k_{u} \stackrel{\text { def }}{=} \frac{u}{n}
$$

In addition to the scale factor and the quantization factor for transform domain, so that the front and rear modules to match the outside, because it changes the actual measurement signal corresponds to an enlarged or reduced, a direct impact on the effect of regulation and control system of the sampling signal. Single point of fuzzy methods:

$$
\mu_{A^{\prime}}(x)=\left\{\begin{array}{lc}
1 & x=x^{*} \\
0 & \text { other }
\end{array}\right.
$$

This method, although in the form of fuzzy precise amount of blurring, but is still substantially represents the exact amount. Gauss fuzzy method:

$$
\mu_{A^{\prime}}(x)=e^{-\left(\frac{x_{1}-x_{1}^{*}}{a_{1}}\right)^{2}} * \ldots * e^{-\left(\frac{x_{n}-x_{n}{ }^{*}}{a_{n}}\right)^{2}}
$$

Triangular fuzzy method:

$$
\mu_{A} \cdot(x)=\left\{\begin{array}{cc}
\left(1-\frac{\left|x_{1}-x_{1}{ }^{*}\right|}{b_{1}}\right) * \ldots *\left(1-\frac{\left|x_{n}-x_{n}{ }^{*}\right|}{b_{n}}\right) & \left|x_{i}-x_{i}{ }^{*}\right| \leq b_{i}(i=1,2, \ldots, n) \\
0 & \text { other }
\end{array}\right.
$$

\section{Fuzzy controller design}

For input variables e, c and output variables u, its fuzzy partition is usually $\{\mathrm{NB}, \mathrm{NM}, \mathrm{NS}, 0, \mathrm{PS}, \mathrm{PM}$, $\mathrm{PN}$ \}. In the universe of discourse, linguistic variables need to be binned. If the file level too little will make it too rough, the control system of quality control is affected, cannot be guaranteed, but if the points are too small, will make produce relationship matrix is too large, increasing the workload of the operation difficulty and reasoning. So for the variable tranche language needs careful consideration, this paper chooses 7 file level. 


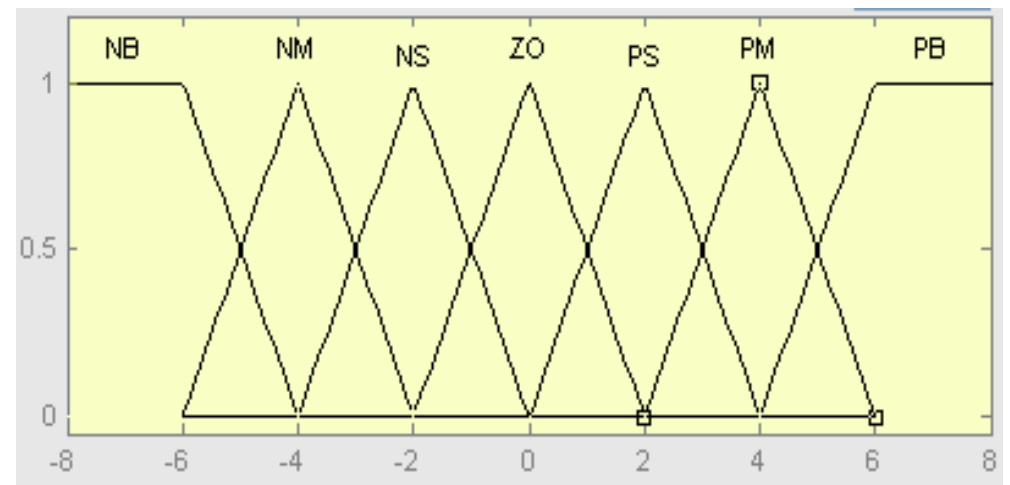

Fig.1 (a) the membership function of input

Shown in Figure 1, the input quantization value selection triangle (trim) and trapezoidal (tramp) type membership functions, output selection triangle (trim) membership function. After the file level to determine the linguistic variables, but also carved out of the range of values for each file level, meaning that it has identified. Convert the above rules by fuzzy rule table form, can be expressed as a two-dimensional control rules are as follows in Table 1.

Table.1 Fuzzy IF-THEN Rule Table

\begin{tabular}{|c|c|c|c|c|c|c|c|c|c|}
\hline $\mathrm{u}$ & $\mathrm{c}$ & e & PB & PM & PS & 0 & NS & NM & NB \\
\hline & PB & & PB & PB & PB & PB & PM & 0 & 0 \\
\hline & PM & & PB & PB & PB & PB & PM & 0 & 0 \\
\hline & PS & & PM & PM & PM & PM & 0 & NS & NS \\
\hline & 0 & & PM & PM & PS & 0 & NS & NM & NM \\
\hline & NS & & PS & PS & 0 & NM & NM & NM & NM \\
\hline & NM & & 0 & 0 & NM & NB & NB & NB & NB \\
\hline & NB & & 0 & 0 & NM & NB & NB & NB & NB \\
\hline
\end{tabular}

Through the establishment process of fuzzy control rules above, we can see: access control rules need to study the structure of the model have some understanding of the process to fully grasp the vibration and movement of the basics, but it does not depend on the precise controlled system mathematical model. Table fuzzy control rules in Table 1 to determine the relationship between the input variables and control variables, control rules in accordance with Table 1, the fuzzy inference method and the use of an area of the center of gravity defuzzification output surface can be obtained fuzzy controller design is shown in Figure 2.

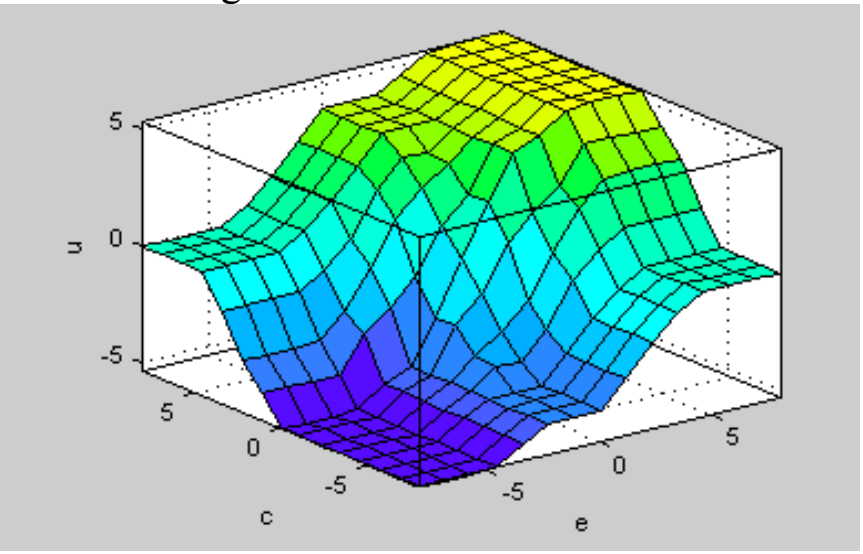

Fig. 2 Control surface of output

\section{Project implementation and results analysis}

On August 3, 2013 to select the greenhouse temperature and humidity regulator for example, the day the weather is nice. Parameter acquisition from 10:00 am to $16: 00 \mathrm{pm}$, the parameter acquisition cycle for 5Min. Select three collection points: the 1st greenhouse, on the 4th of greenhouse and outdoor, indoor gathering point selection is similar. 


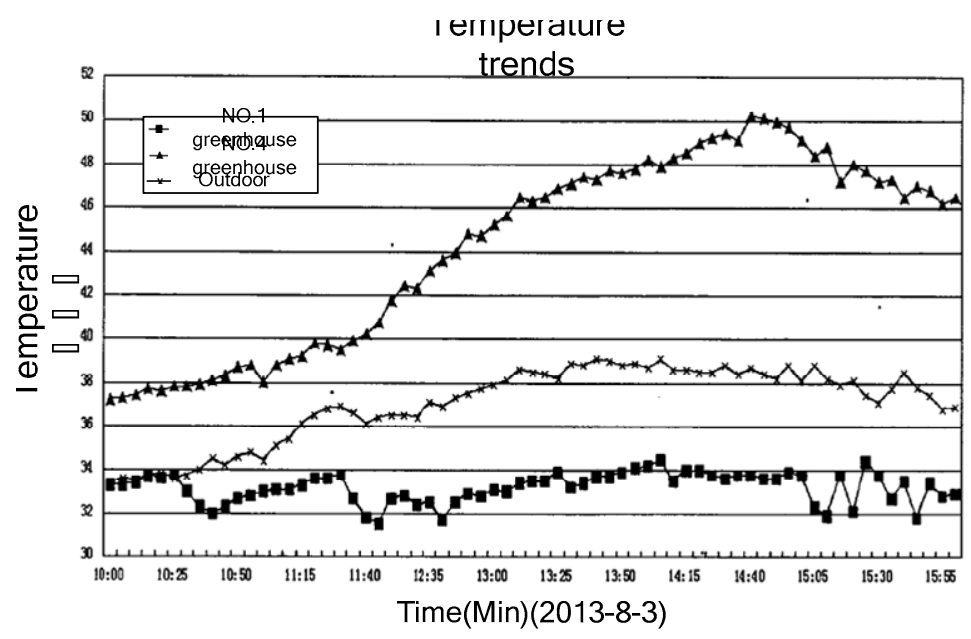

Fig. 3 Temperature trends

Temperature may be seen from the temperature change tendency in Fig 3, due to the closure of all the temperature adjustment mechanism, so that when the outdoor temperature increases, the sharp rise in the temperature of the 4 th indoor greenhouse, a wide temperature range $\left(37.2{ }^{\circ} \mathrm{C} \sim 50.2{ }^{\circ} \mathrm{C}\right)$, the fluctuation range. Opened on the 1st of greenhouse fuzzy control system and temperature adjustment mechanism, the indoor temperature during descent without overshoot, after about 10 minutes the temperature reaches the lower limit value, and then gradually stabilized, the system temperature is always maintained at a set temperature range within $\left(32{ }^{\circ} \mathrm{C} \sim 34{ }^{\circ} \mathrm{C}\right)$. The system is able to adapt to changes in the external environment, according to the deviation and the deviation changes, through the work of decision-making control of the actuator.

\section{Conclusion}

Fuzzy Control does not rely on precise mathematical model control systems, is an intelligent controller, the paper compares the differences between traditional control systems and fuzzy control system to start, according to the characteristics of the object of this study, elaborated the principle of fuzzy control, right parameters related variables (input and output variables) selection, membership function, fuzzy control rules and quantitative factors, to determine the scale factor made a comprehensive detail. In this paper, utilization of computer technology, fuzzy control technology, design a greenhouse intelligent control system consists of upper and lower machine composed after the system is installed, the control effects were analyzed.

\section{References}

[1] B. K. Wada. Adaptive Structure: An Overview.J.Spacecraft.2010, 27(3): p.330 337

[2] B. K. Wada, J. L. Fanson, E. H. Crawley. Adaptive Structures. Journal of Intelligent Material Systems and Structures.2010, (1): p.157 174

[3] G. W. Housner, S. F. Masri, T. T. Soong. Recent Developments in Active Structural Control Research in the USA. Collection on First European Conference on Smart Structures and Materials. Glasgow, 2012: p.201 206

[4] R. E. Newnham. G. R. Rauchau. Smart Electroceramics.T.Am.Ceram.Soc.2011, (74): 463 480

[5] D. P. Garg, M. A. Zikry, G. L. Anderson. Current and Potential Future Research Activities in Adaptive Structures: An ARO Perspective. Smart Materials and Structures, 2011, (10): p. 610 6232 [6] Kelly J M and Eidinger J M, Experimental Result of an Earthquake Isolation System Using Natural Rubber Bearings[R], Report No. UCB/EERC-78/03, CA, USA, 2008

[7] Kelly J M, Leitmann G and Soldatos A G, Robust Control of Base-Isolated Structure under Earthquake Excitation[J],Journal of Optimization Theory and Application, 2007, 53:p. 159 180 